\title{
Mathematik als Sport
}

\author{
Ein Gespräch mit Lisa Sauermann
}

\author{
Günter M. Ziegler
}

\begin{abstract}
Lisa Sauermann, Jahrgang 1992, wurde schon als Schülerin des Dresdner Martin-Andersen-Nexö-Gymnasiums bekannt, weil sie auf der Internationalen Mathematik-Olympiade IMO regelmäßig Medaillen für Deutschland holte (wir berichteten regelmäßig). Als sie 2011 als einzige Teilnehmerin die volle Punktzahl erreichte und damit eine Goldmedaille errang, war sie die erfolgreichste IMO-Teilnehmerin aller Zeiten. Ein Jahr später wurde ihr Rekord von Teodor von Burg aus Serbien gebrochen. Nach dem Abitur studierte Lisa Sauermann Mathematik in Bonn. Sie promovierte 2019 bei Jacob Fox an der Stanford University. Ihre Dissertation wurde dieses Jahr mit dem Richard-Rado-Preis der DMV-Fachgruppe Diskrete Mathematik ausgezeichnet - Anlass für die Mitteilungen, mit der begabten Nachwuchsmathematikerin zu sprechen.
\end{abstract}

Vielen unserer Leserinnen und Leser bist du als erfolgreichste Teilnehmerin an Mathe-Schülerwettbewerben der 2oooer Jahre bekannt. Daher wüssten wir gerne als erstes, wie das alles so anfing mit der Mathematik, im Elternhaus, in der Schule, mit den Wettbewerben usw.

Meine Eltern sind keine Mathematiker. Aber mein Vater ist Ingenieur und meine Mutter Physikerin. Beide konnten also Mathe und haben mich als Kind darin unterstützt. Als ich dann in der 4. Klasse war, hat meine Mutter organisiert, dass ich an der Matheolympiade teilnehme, die damals eigentlich erst ab Klasse 5 war. Das war eine tolle Erfahrung und hat mich sehr motiviert.

Weil es dann auch erfolgreich war?

Ja, aber nicht nur deshalb. Die erste Stufe ist ja sowieso nur eine Hausaufgabe. Für die zweite Stufe hatte ich dann an der Grundschule einen Tag schulfrei, um an der Matheklausur an der anderen Schule teilnehmen zu können und die dritte Stufe war dann schon regional, also auf Bezirksebene damals, auch ein schönes Erlebnis. Ich glaube, ich habe damals einen dritten Preis gemacht, also nichts Bombastisches. [lacht]

Hast du Geschwister?

Ja, ich hab eine jüngere Schwester.

War deine Schwester bei den Mathe-Wettbewerben auch dabei?

Ja, war sie. Aber es war für sie eine eher schwierige Situation: In der 5 . Klasse habe ich wieder mitgemacht und war noch erfolgreicher und hab in der dritten Stufe gewonnen. Das war natürlich super motivierend für mich. Aber das klingt jetzt so, als ob nur der Erfolg motivierend war. Natürlich haben mir die Aufgaben Spaß gemacht, aber ich mochte es als Kind auch zu den Wettbewerben hinzufahren, in den Landheimen zu übernachten und mit anderen Leuten zusammen zu sein. Und als ich in der 6. Klasse das erste Mal bei der Bundesrunde der
Mathematik-Olympiade dabei war, bin ich für die Achtklässler angetreten und hab einen ersten Preis geholt. Da konnte man dann vielleicht schon sagen, das war jetzt ungewöhnlich. Für meine Schwester wurde es dann natürlich schwierig, weil sie immer so in meinem Schatten stand, das macht dann ja nicht so viel Spaß. Sie war schon auch gut, nur eben nicht auf dem gleichen Level. Sie hat dann später angefangen sich mehr für Physik zu interessieren, hat das auch studiert und promoviert jetzt sogar in Physik.

Was ja auch nicht etwas ganz anderes ist.

Genau! Meine Schwester ist auf alle Fälle sehr talentiert in den mathematisch-naturwissenschaftlichen Fächern. Die Mathematik war nur schon vergeben und es macht dann ja nicht so viel Spaß, wenn man im Schatten der älteren Schwester steht. Und da hat sie sich eben für Physik entschieden.

Gab es da Lehrer, die dir besonders geholfen haben? Du warst ja auf dem Martin-Andersen-Nexö-Gymnasium, einer Spezialschule in Dresden.

Ja, es gab da eine Mathe-AG. Die ging eigentlich schon in der 4. Klasse los, um die potentiellen Kandidaten für die 5. Klasse anzuwerben. Und die ging in der 5 . Klasse natürlich weiter. Und später gab es dann ein Mathetraining an der TU Dresden für die, die gut in der Matheolympiade abgeschnitten haben. Das hat mir sehr geholfen ...

... im Grunde alles noch Förderstrukturen aus DDRZeiten, oder?

Ja. Ich denke auch, dass das einer der Gründe ist, warum Sachsen zumindest früher - ich weiß nicht genau wie es heute ist - durchaus mit bevölkerungsreicheren Bundesländern wie Bayern oder Nordrhein-Westfalen mithalten konnte, weil in Sachsen Talente schon früh gefunden und gefördert wurden. Die Matheolympiade ist in Sachsen auch viel bekannter, so dass viel mehr Schüler davon erreicht werden. Für mich war damals toll, dass man schon 


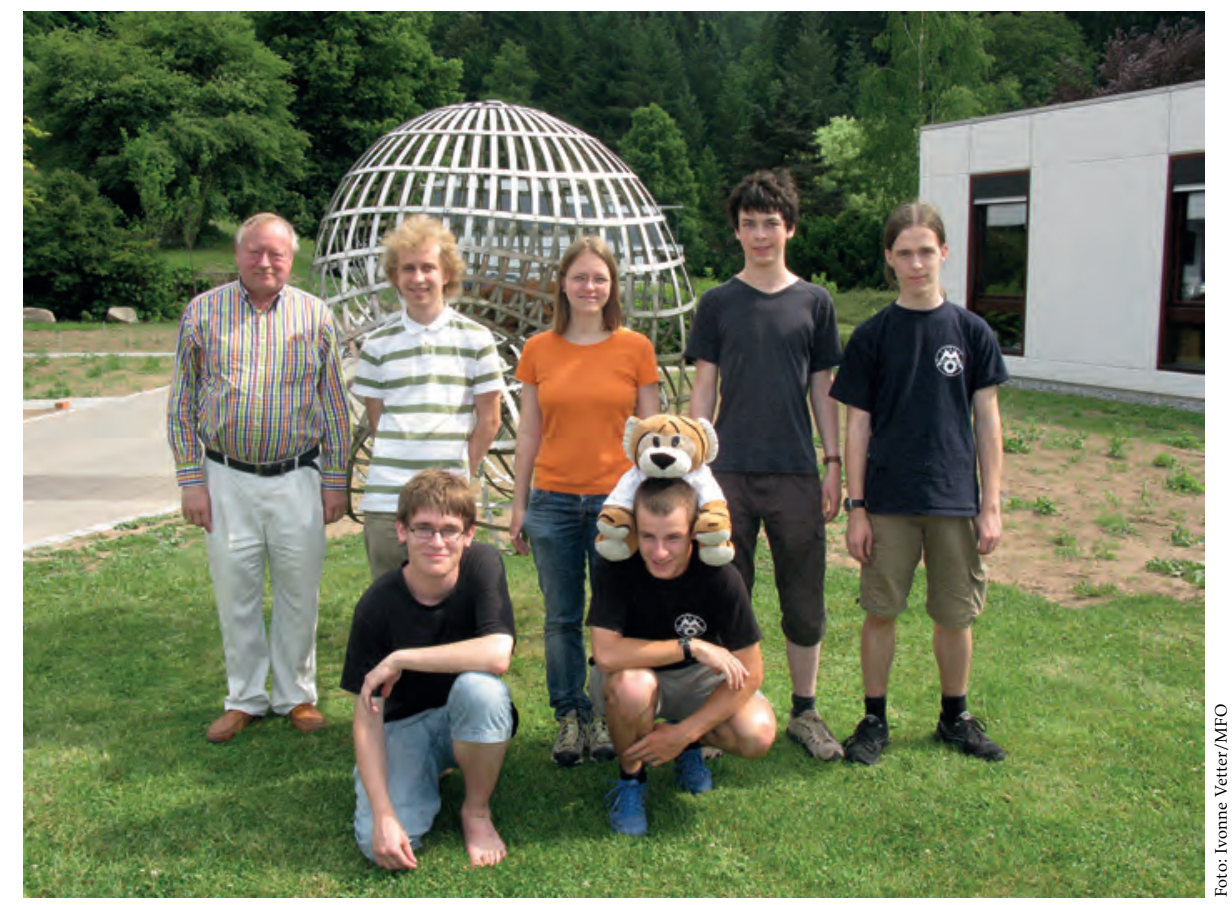

Trainings- und Abschluss-Seminar für die IMO 2011 am MFO

Stehend: Hans-Dietrich Gronau, Florian Schweiger, Lisa Sauermann, Bernhard Reinke, Marius Graeber; knieend: Achim Krause und Michael Schubert

relativ jung, also ab der 5 . Klasse, auch mal woanders hin eingeladen wurde. Und es gibt in Sachsen ja auch das Landesseminar zur Vorbereitung und Auswahl des Bundesrundenteams, eine ganze Woche lang. Da war ich das erste Mal in der 6. Klasse - das war eine tolle Woche! All die anderen Schüler zu treffen war - jedenfalls für mich - total motivierend und hat großen Spaß gemacht; nicht, weil man die ganze Zeit Mathe gemacht hat, sondern weil man auch Spiele gespielt hat und so weiter.

Also ein bisschen Mathematik als Pfadfinder-

Erlebnis...

Genau!

Hat nur gefehlt, dass es Zeltlager gab.

Ja, Zelte gab es nicht, wir waren immer in Jugendherbergen. Aber sonst wahrscheinlich Ja.

Das heißt, Mathematik nimmt man dann eher wahr als eine Art sportliche Disziplin, oder? Andererseits ist man ja doch immer im Wettbewerb und es ist das Problemlösen, das die eigentliche Kunst ist, die vorgeführt und gemacht wird.

Ja, das ist schon eine ganz andere Mathematik als die echte Mathematik. Das halten ja auch viele der Matheolympiade vor, dass es keine richtige Mathematik ist. Und ich denke, das stimmt vollkommen. Das heißt natürlich nicht, dass es eine schlechte Sache ist. Es ist halt eine Art Sport, die so ein bisschen mit echter Mathematik zu tun hat. Die Mathe-Olympiade-Aufgaben sind ja schon speziell insofern, dass sie mit begrenzten Mitteln auskommen und sich die Tricks oft wiederholen.
Stellt sich die Frage ob die Schulmathematik, also der Stoff von der 1. bis zur 12./13. Klasse, Mathematik ist.

Also da sind die Aufgaben der Mathe-Olympiade sicher näher dran an echter Mathematik. Denn die MatheOlympiade-Aufgaben sind ja immerhin mit Beweisen. Das ist ja doch der Kern dessen, was Mathematik ausmacht, und in vielen Bundesländern wird ja auch in der Oberstufe überhaupt nicht mehr bewiesen und in der Mathe-Olympiade schon.

Nun, das Beweisen ist dann ja die Form in die alles mündet. Es ist ja so, dass man sowohl im Studium als auch in der Schule mit abstrakten Strukturen klarkommen muss. Und man muss eine gewisse Präzision entwickeln und ein Gefühl dafür, was sein kann und was nicht plausibel ist. Insofern ist es ja noch mehr als nur die Konstruktion von Beweisen ...

Ja, das stimmt. Aber als ich auf der Schule war, wurde doch viel Wert darauf gelegt gewisse Rechenalgorithmen anzuwenden. Zum Beispiel „Wie finde ich die Extremwerte einer Funktion?" Da ging man durch das Rechenschema durch, aber es wurde überhaupt nicht erklärt, warum wir da jetzt die Nullstellen der Ableitung nehmen. Die meisten Schüler konnten das Schema anwenden, hatten aber überhaupt keine Ahnung, warum sie da jetzt die Ableitung nehmen. Und in der „echten Mathematik“ ist es ja so, dass man gerade nicht weiß, was man machen muss. Da muss man ja kreativ sein. Und bei den MatheOlympiade-Aufgaben muss man auch kreativ sein, man weiß am Anfang auch nicht, wie es geht. Insofern gibt es schon Parallelen in Bezug auf die Herangehensweise. 
An welchen Wettbewerben hast du noch teilgenommen?

Am Bundeswettbewerb Mathematik habe ich in der 7. Klasse das erste Mal teilgenommen, glaube ich. Aber ich weiß, dass ich in der 8 . Klasse das erste Mal Bundessiegerin geworden bin.

Also auch ziemlich steil nach oben! Und nach fünf oder sechs Bundessiegen muss man ja die 3. Runde gar nicht mehr machen, oder?

Ja, wenn man es einmal zum Bundessieg geschafft hat, ist es danach deutlich einfacher.

Was dann international sichtbar wird, ist ja die Internationale Mathematik-Olympiade, die IMO. Die hast du fünfmal gemacht und immer gewonnen mit einmal Silber und viermal Gold. Ist das auch so, dass wenn man die einmal gewonnen hat, dann wird es locker? Oder wird der Druck dann immer größer, weil alle von einem so viel erwarten?

Es ist auf alle Fälle nicht so einfach wie beim Bundeswettbewerb, wo man dann einfach eine Stufe überspringt. Es wird schwieriger insofern, dass der Druck zunimmt, aber es wird auch leichter, weil man in der Vergangenheit schon mehr trainiert hat. Das Training über viele Jahre hinweg zahlt sich dann aus. Aber es ist jedes Jahr auch viel Glück dabei, ob einem die Aufgaben nun gerade liegen oder nicht. Und ob man gerade die richtige Idee hat oder nicht.

Der Maßstab wird dann ja auch jedes Jahr neu adjustiert, je nachdem wie schwierig die Aufgaben waren oder sind ... Und manchmal ist es dann eben leichter oder schwieriger.

Ja, aber es kommt ja auch immer etwas auf die Auswahl an. Dadurch dass es immer sechs Aufgaben gibt, sind verschiedene Gebiete unterschiedlich oft abgedeckt. Wenn einem jetzt zum Beispiel Zahlentheorie mehr liegt als Kombinatorik, ist es natürlich eine Glücksfrage, ob es zwei Aufgaben aus der Zahlentheorie gibt oder zwei aus der Kombinatorik. Die Allgemeine Schwierigkeit trifft alle, aber jeder hat ja noch eigene Stärken oder Schwächen ...

Und wo waren damals deine Stärken und Schwächen?

Also Geometrie und Kombinatorik waren meine Stärken. Algebra und Zahlentheorie waren eher meine Schwächen. Vor allem Ungleichungen waren meine größte Schwäche. Ich hatte Glück, dass in den fünf Jahren nur eine Ungleichung drankam. [lacht]

\section{Und die hat dann zur Silbermedaille geführt?}

Nein. Die Ungleichungsaufgabe kam in meinem zweiten Jahr und ich hab' sie nicht gelöst. Aber es hat dann immer noch für Gold gereicht. Ich hatte halt alle anderen Aufgaben.
Was ist denn das besondere Talent der Lisa Sauermann gewesen? Genialität oder harte Arbeit oder eine besondere Konzentrationsfähigkeit, die keiner schlagen konnte, oder alles zusammen?

Ich weiß nicht. Ich hab schon hart gearbeitet, aber viele andere auch. Ich würde jedenfalls nicht sagen, dass ich so viel härter gearbeitet habe als alle anderen. Und Konzentrationsfähigkeit? Ich weiß nicht. Ich glaube, es hat mir Spaß gemacht und so war ich sehr motiviert. Und ich hab es so viele Jahre lang gemacht und der Erfolg motiviert natürlich auch, aber genau weiß ich es nicht.

Hast du zufällig den Roman gelesen, der gerade rauskam, von Patrick Hofmann, Nagel im Himmel? In dem der Held ziemlich am Anfang auf die Internationale Mathematik-Olympiade in Montreal fährt? Und teils aus Schusseligkeit, teils aus Undiszipliniertheit keine Medaille bekommt?

Nein, kenne ich nicht. Das Wichtigste für mich an der IMO war auch nicht die Punktzahl oder die Medaille, sondern dabei zu sein. Das war einfach eine tolle Erfahrung!

Warst du eigentlich 2009 in Bremen dabei, als ich bei der Preisverleihung das nord- und das südkoreanische Team zusammen auf die Bühne gerufen habe?

Ja, da war ich dabei und ich erinnere mich auch, wie die auf der Bühne standen ...

Gibt es eine Mathematik Olympiade, an die du besonders gerne zurückdenkst?

Also es waren alle toll. Aber die in Bremen war schon besonders für mich, weil ich so viele Leute kannte. Viele der guides waren aus der Mathematik-Olympiade-Szene. Also Leute, die in den Vorjahren an einer IMO teilgenommen haben oder in der Auswahl waren. Und dann natürlich die ganzen Koordinatoren. Also das war schon toll, all diese Leute auf dem Campus...

Das ist dann wie eine Weltmeisterschaft im eigenen Land, oder?

Ja.

Hast du aus der Mathematik-Olympiade-Zeit eine Lieblingsaufgabe?

Ah, das ist eine schwere Frage! Also vielleicht die Grashüpfer-Aufgabe in Bremen ... und die WindmühlenAufgabe in Amsterdam [www.imo-official.org/problems. aspx]. Ja, die beiden würde ich sagen, also zwei Favoriten ...

Gab's bei der Grashüpfer-Aufgabe nicht nur ganz wenige Lösungen?

Ich denke, es gab mindestens drei, weil es ja zwei Leute mit voller Punktzahl gab und ich hatte 41 [Punkte].

Wo sind eigentlich die Lösungen von Lisa Sauermann abgeblieben? 
Die Aufgaben der 50. IMO 2009 in Bremen

1. Es seien $n$ und $k$ positive ganze Zahlen mit $k \geq 2$. Ferner seien $a_{1}, \ldots, a_{k}$ paarweise verschiedene ganze Zahlen aus der Menge $\{1, \ldots, n\}$ derart, dass $n$ die Zahl $a_{i}\left(a_{i+1}-1\right)$ für jedes $i=1, \ldots, k-1$ teilt. Man zeige, dass dann $n$ die Zahl $a_{k}\left(a_{1}-1\right)$ nicht teilt.

(Australien)

2. Es sei $A B C$ ein Dreieck mit Umkreismittelpunkt $O$. Es seien $P$ und $Q$ innere Punkte der Seiten $C A$ und $A B$. Ferner seien $K, L$ und $M$ die Mittelpunkte der Strecken $B P, C Q$ bzw. $P Q$. Der Kreis $\Gamma$ gehe durch $K, L$ und $M$. Die Gerade $P Q$ sei Tangente an den Kreis $\Gamma$. Man zeige, dass $|O P|=|O Q|$ gilt. (Russland)

3. Es sei $s_{1}, s_{2}, s_{3}, \ldots$ eine streng monoton wachsende Folge positiver ganzer Zahlen derart, dass die beiden Teilfolgen

$$
s_{s_{1}}, s_{s_{2}}, s_{s_{3}}, \ldots \quad \text { und } s_{s_{1}+1}, s_{s_{2}+1}, s_{s_{3}+1}, \ldots
$$

jeweils arithmetische Folgen sind. Man zeige, dass $s_{1}, s_{2}, s_{3}, \ldots$ ebenfalls eine arithmetische Folge ist.

4. Es sei $A B C$ ein Dreieck mit $|A B|=|A C|$. Die Innenwinkelhalbierenden der Winkel $B A C$ und $C B A$ schneiden die Seiten $B C$ und $A C$ in den Punkten $D$ bzw. $E$. Es sei $K$ der Inkreis-

\section{Die Aufgaben der 52. IMO 2011 in Amsterdam}

1. Für jede Menge $A=\left\{a_{1}, a_{2}, a_{3}, a_{4}\right\}$ von vier paarweise verschiedenen positiven ganzen Zahlen, deren Summe mit $a_{1}+a_{2}+a_{3}+a_{4}$ mit $s_{A}$ bezeichnet werde, sei $n_{A}$ die Anzahl der Paare $(i, j)$ mit $1 \leq i<j \leq 4$, für die $a_{i}+a_{j}$ die Zahl $s_{A}$ teilt. Man bestimme unter all diesen Mengen $A$ diejenigen, für die $n_{A}$ maximal ist.

(Mexiko)

2. Sei $\mathcal{S}$ eine endliche Menge von mindestens zwei Punkten in der Ebene. Dabei wird angenommen, dass keine drei Punkte von $\mathcal{S}$ kollinear sind. Als Windmühle bezeichnen wir einen Prozess der folgenden Art. Wir starten mit einer Geraden $\ell$, die genau einen Punkt $P \in \mathcal{S}$ enthält. Die Gerade $\ell$ wird im Uhrzeigersinn um den Drehpunkt $P$ so lange gedreht, bis sie zum ersten Mal auf einen weiteren Punkt aus $\mathcal{S}$, der mit $Q$ bezeichnet sei, trifft. Die Gerade wird weiter im Uhrzeigersinn mit $Q$ als neuem Drehpunkt gedreht, bis sie wieder auf einen Punkt aus $\mathcal{S}$ trifft. Dieser Prozess wird unbegrenzt fortgesetzt. Man beweise, dass für geeignete Wahl eines Punktes $P \in \mathcal{S}$ und einer Ausgangsgeraden $\ell$, die $P$ enthält, die resultierende Windmühle jeden Punkt aus $\mathcal{S}$ unendlich oft als Drehpunkt hat.

(Vereinigtes Königreich)

3. Sei $f: \mathbb{R} \rightarrow \mathbb{R}$ eine Funktion, die die Bedingung

$$
f(x+y) \leq y f(x)+f(f(x))
$$

für alle reellen Zahlen $x$ und $y$ erfüllt.

Man beweise, dass $f(x)=0$ für alle $x \leq 0$ gilt. (Weißrussland)

Die bekommt man ja zurück, also liegen die wohl irgendwo bei meinen Eltern im Keller...

\section{Nach der Olympiadezeit bist du dann zum Studium nach Bonn gegangen, warum dorthin?}

Weil alle meine Freunde von der Mathematik-Olympiade dahin gegangen sind. Das wechselt sicher immer mal, aber damals gingen alle dorthin. [lacht] mittelpunkt des Dreiecks $A D C$. Ferner sei $\Varangle B E K=45^{\circ}$. Man bestimme alle möglichen Werte von $\Varangle B A C$.

(Belgien)

5. Man bestimme alle Funktionen $f$, die auf der Menge der positiven ganzen Zahlen definiert sind und nur positive ganze Zahlen als Werte annehmen, so dass es für alle positiven ganzen Zahlen $a$ und $b$ ein nicht entartetes Dreieck mit Seitenlängen

$$
a, f(b) \text { und } f(b+f(a)-1)
$$

gibt.

(Ein Dreieck heißt nicht entartet, wenn seine Eckpunkte nicht kollinear sind.)

(Frankreich)

6. Es seien $n$ eine positive ganze Zahl, $a_{1}, a_{2}, \ldots, a_{n}$ paarweise verschiedene positive ganze Zahlen und $M$ eine Menge von $n-1$ positiven ganzen Zahlen, die nicht die Summe $s=a_{1}+a_{2}+\cdots+a_{n}$ als Element enthält. Ein Grashüpfer springt längs der reellen Zahlengerade. Er startet im Nullpunkt und vollführt $n$ Sprünge nach rechts mit Längen $a_{1}, a_{2}, \ldots, a_{n}$ in beliebiger Reihenfolge. Man zeige, dass der Grashüpfer seine Sprünge so anordnen kann, dass er nie auf einem Punkt aus $M$ landet.

(Russland)

4. Sei $n>0$ eine ganze Zahl. Gegeben seien eine Balkenwaage und $n$ Gewichtsstücke mit den Gewichten $2^{0}, 2^{1}, \ldots, 2^{n-1}$. Wir sollen jedes der $n$ Gewichtsstücke, eines nach dem anderen, so auf die Waage legen, dass die rechte Schale zu keinem Zeitpunkt schwerer als die linke ist. In jedem Zug wählen wir ein Gewichtsstück aus, das zu diesem Zeitpunkt noch nicht auf die Waage gelegt wurde und legen es entweder auf die linke oder die rechte Schale, bis alle Gewichtsstücke verwendet worden sind.

Man bestimme die Anzahl derartiger Folgen mit $n$ Zügen.

5. Sei $f$ eine Funktion, die die Menge der ganzen Zahlen in die Menge der positiven ganzen Zahlen abbildet. Für je zwei ganze Zahlen $m$ und $n$ sei die Differenz $f(m)-f(n)$ durch $f(m-n)$ teilbar.

Man beweise für alle ganzen Zahlen $m, n$ mit $f(m) \leq f(n)$, dass $f(n)$ durch $f(m)$ teilbar ist.

(Iran)

6. Es seien $A B C$ ein spitzwinkliges Dreieck mit dem Umkreis $\Gamma$ und $\ell$ eine Tangente an $\Gamma$. Ferner seien $\ell_{a}, \ell_{b}$ und $\ell_{c}$ die Geraden, die durch Spiegelungen von $\ell$ an den Geraden $B C, C A$ bzw. $A B$ entstehen.

Man beweise, dass der Umkreis des Dreiecks, das von den Geraden $\ell_{a}, \ell_{b}$ und $\ell_{c}$ gebildet wird, den Kreis $\Gamma$ berührt. (Japan)

Und da ist man dann praktisch im Team hin zum Studieren gegangen ...?

Ja, wenn alle dahin gehen, dann muss das ja gut sein und wenn alle Freunde da hingehen, dann macht es ja auch mehr Spaß. Und es ist dann ja auch ein sich selbst verstärkender Effekt. Ich weiß nicht, ob es immer noch Bonn ist, ein paar Jahre zuvor sind alle nach München gegangen ... 


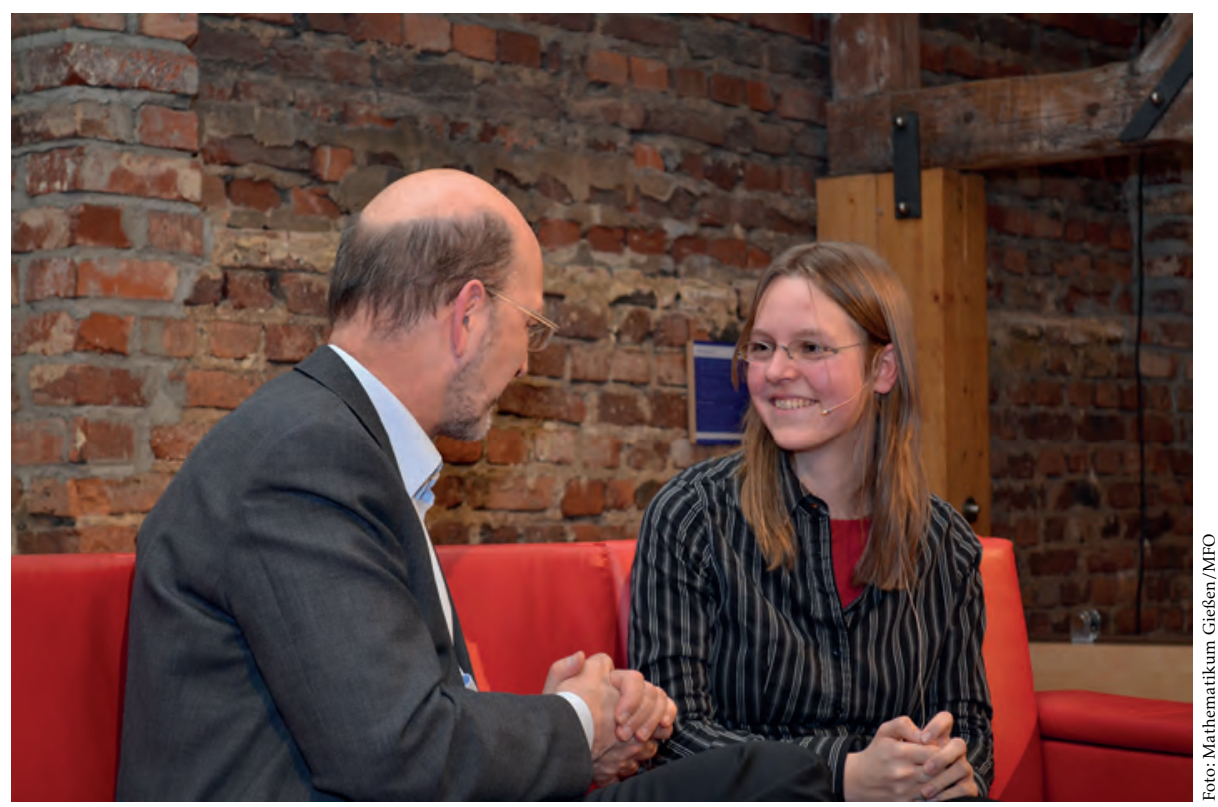

Lisa Sauermann bei Albrecht Beutelspacher auf „Beutelspachers Sofa“ Mathematikum Gießen 2012

Dann zum Einstieg ins Studium. Studiumsmathematik ist ja etwas anderes als Schulmathematik und auch als Wettbewerbsmathematik. War der Einstieg schwierig?

Also ich fand die ersten Monate schon schwierig, aber das lag auch an den persönlichen Veränderungen. Wenn man lange bei seinen Eltern wohnt, wo immer für einen gekocht wird ... Und dann zieht man aus und muss sich um alles selber kümmern, dann ist das, denke ich, auch normal, dass das eine Umstellung ist. Ich hab damals in einer WG gewohnt, da lief auch nicht immer alles rund, auch wenn es alles in allem eine gute Zeit war. Und dann hab ich noch - und das war ein Fehler - zu viele Vorlesungen gemacht am Anfang. Ich glaub ich hab mit sechs Vorlesungen angefangen. Das ist zu viel, auf jeden Fall.

Ja, das kommt mir irgendwie bekannt vor, als Fehler auch ... Und du hast zu der Zeit Kochen gelernt, oder wer hat gekocht, in der WG?

Wir haben uns abgewechselt und ich hab auch etwas Kochen gelernt.

Und an der Uni Bonn hast du dich auf Diskrete Mathematik konzentriert?

Nein, gar nicht. In Bonn ist das ja ein separates Institut. Da hab ich überhaupt keine Vorlesungen gemacht, zu der Zeit. Das hab ich damals ein bisschen verschlafen oder verpasst oder mich einfach für andere Sachen interessiert. Ich hab damals hauptsächlich Vorlesungen in Algebra und Algebraischer Topologie - und Logik und Mengenlehre so ein bisschen - gemacht. Und ich hatte dann den Plan, dass ich mich Richtung Algebra und Algebraische Geometrie spezialisieren würde und das ist dann am Ende anders gekommen. Aber das hat sich erst geändert, als ich in Stanford war.
Das ist ja ein typisch Bonner Plan. Wenn man hochbegabt nach Bonn kommt, muss man am Ende ShimuraVarietäten untersuchen?

Bis zu den Shimura-Varietäten bin ich nicht gekommen, aber ich war auf dem Weg dahin, ja. [lacht]

Und wie ist das dann "schiefgegangen“?

Ich bin dann nach dem Bachelor nach Stanford gegangen. Ursprünglich hatte ich ein Jahresstipendium vom DAAD für ein Jahr in den USA. Dann war das aber so, dass man nach den Regeln des DAAD regulär eingeschrieben sein musste, und nicht als Besuchsstudent. Aber wenn man schon den Bachelor hat, kann man sich in den USA nur als Promotionsstudent einschreiben. Masterprogramm in Mathe kannten die gar nicht in Stanford und so hab ich mich für das PhD-Programm beworben und gesagt, dass ich nicht weiß, ob ich länger als ein Jahr bleiben kann. Das war für Stanford aber OK, weil ja der DAAD für ein Jahr bezahlt hat. Und nach einem Jahr hab ich mich dazu entschieden für den ganzen $\mathrm{PhD}$ zu bleiben.

Im ersten Jahr in Stanford hab ich hauptsächlich Algebraische Geometrie gemacht und die allgemeinen Kurse, die man als erstes machen muss. Und in meinem zweiten Jahr ist Jacob Fox nach Stanford gekommen und dann hab ich aus Spaß eine Kombinatorik-Vorlesung bei ihm angehört. Weil ich das in der Olympiade immer cool fand und da wollte ich dann doch mal eine richtige Vorlesung dazu hören und das hat mir dann so gefallen, dass ich meine Richtung geändert habe.

Das wäre dann auch meine Frage: Sucht man sich erst ein Thema oder ein Gebiet oder sucht man sich erst einen Betreuer oder was kommt da zuerst? 
In dem Fall war die schwierige Entscheidung für mich zwischen Algebraischer Geometrie und Kombinatorik. Und es war klar, würde ich Kombinatorik machen, dann wäre das mit Jacob Fox. Wenn ich Algebraische Geometrie gemacht hätte, wäre es mit Ravi Vakil gewesen.

Mit Ravi Vakil kann man bestimmt auch ganz wunderbar zusammenarbeiten.

Ja, der ist super! Der Grund, weshalb ich mich am Ende für Kombinatorik entschieden habe, war, dass ich dachte, es liegt mir mehr.

Am Ende sieht das ja auch sehr geradlinig aus, weil man die Kombinatorik schon aus der Wettbewerbsmathematik kennt. Und es kann ja auch sein, dass die Begeisterung schon aus der Zeit stammt.

Ja, ich hatte auf alle Fälle schon eine Begeisterung für Kombinatorik. Aber wie du schon vorhin sagtest, wenn man in Bonn ist, macht man eben Algebra. [lacht]

In deiner Dissertation sind ja durchaus algebraische Methoden drin, Partitionstechniken und so weiter.

Ja. Ich hab dann natürlich, nachdem ich mich für Kombinatorik entschieden habe, auch nach Projekten gesucht, in die ich meinen Hintergrund in Algebra und Algebraischer Geometrie einbringen kann. Und mein Betreuer Jacob Fox hat mir auch gezielt Projekte vorgeschlagen, die in die Richtung gingen.

Und ist da jetzt auch ernsthafte algebraische Geometrie drin in der Dissertation? Oder würden Algebraische Geometer das für läppisch halten?

Die würden das eher für läppisch halten. Und es betrifft ja auch nur eines der Projekte. In den anderen sind ja nur ein paar Polynome drin, das ist doch sehr grundlegende Algebra. In Bezug auf das eine Projekt, das etwas Algebraische Geometrie benutzt: Ich denke, die Algebraischen Geometer würden die Algebraische Geometrie darin langweilig finden. Also es ist reelle Algebraische Geometrie, was für die Algebraischen Geometer etwas exotisch ist, die meisten arbeiten ja nicht mit reellen Räumen. Natürlich gibt es Bücher über reelle Algebraische Geometrie, aber die konkreten Aussagen, die ich brauchte, die habe ich nirgends gefunden. Deshalb hat das Paper jetzt einen Anhang, in dem diese Aussagen dann bewiesen werden. Aber der Anhang ist jetzt nicht so gedacht, dass das jemand algebraisch interessant oder toll finden muss, sondern nur der Vollständigkeit halber. Denn diese Aussagen in reeller Algebraischer Geometrie gehen als Black Box in das kombinatorische Argument ein.

Also ich finde es ja immer interessant, wenn man Hilfsmittel aus einem Gebiet - in diesem Fall der reellen Algebraischen Geometrie - nimmt und damit Probleme ganz woanders, in diesem Fall in der Kombinatorik, löst.
Ja. Und ich denke, dass das, was ich da in mehreren Jahren im Bereich Algebraischer Geometrie gelernt habe, geholfen hat.

Freeman Dyson, der kürzlich gestorben ist, hat diesen Aufsatz über Frösche und Vögel geschrieben [siehe Mitteilungen 28-1], in dem er die Mathematiker beschreibt als einerseits die Problemlöser, die Frösche, die bodennah arbeiten und das Konkrete machen, und dann die Vögel, die Theoriebauer, die die gesamte Landschaft überblicken, aber sich um die Details nicht so viele Sorgen machen können, weil sie viel zu weit davon weg sind. Frage A: Machen diese Kategorien Sinn? Frage B: Wenn ja, Frosch oder Vogel?

Also ich kann mir vorstellen, dass die Kategorien in vielen Bereichen der Mathematik Sinn machen, aber ich weiß jetzt nicht, ob ich in der extremalen Kombinatorik so einen Unterschied sehe. In Kombinatorik oder zumindest in extremaler Kombinatorik ist es nicht so, dass man dieselben Theoriegebäude hat wie in anderen Gebieten. In Algebraischer Geometrie muss man ja viele Jahre lang Vorlesungen hören, bis man überhaupt die Fachbegriffe versteht, die in einem aktuellen Paper stehen. Und so ist es in Kombinatorik überhaupt nicht. Wenn man eine Einführungsvorlesung in Kombinatorik gehört hat, dann kann man vielleicht nicht direkt jedes Paper lesen, aber wenn jemand einem eine halbe Stunde lang die Begriffe erklärt, die in genau dem Paper vorkommen, dann würde man zumindest das Hauptresultat des Papers verstehen. Insofern ist es vielleicht so, dass die Unterscheidung zwischen Fröschen und Vögeln für extremale Kombinatorik nicht so viel Sinn ergibt. Vielleicht sind wir auch alle Frösche in extremaler Kombinatorik, das kann auch sein.

Also die Vorurteile gegenüber Diskreter Mathematik, die es früher gab, die kamen schon auch daher, dass Grothendieck nichts dazu beigetragen hat und es die Kategorieansätze in dem Sinne nicht gibt, sondern es ein Problemlöse-Fach ist.

Ja. Ich wollte auch nicht sagen, dass es in [extremaler Kombinatorik] keine Theorie gibt, sondern dass sie nicht ganz so abstrakt auf so vielen Stufen übereinander aufbaut; also, dass man nicht jahrelang Definitionen lernen muss bis man die Aussage eines aktuellen Papers versteht.

Vielleicht noch etwas zum Arbeitsstil von Lisa Sauermann ... Ist das, was du ablieferst, eigentlich von Anfang an präzise und perfekt oder fängt man da eher an mit Skizzen und Versuchen und arbeitet die dann aus? Und muss schauen, dass man die Sachen irgendwann solide und vollständig macht, dass es dann stimmt?

Also wenn man da erst mal so eine Idee hat, dann ist die zumindest bei mir noch nicht solide und perfekt. Aber es gibt da, denke ich, unterschiedliche Herangehensweisen. Manche Leute tippen das schon mal ab ... und verbessern das dann.

Ich persönlich bin so langsam im Tippen, dass ich möglichst lange auf Papier arbeite, bis es halbwegs stimmt. 
Und wenn ich es dann abtippe, dann ist es hoffentlich schon richtig.

Das vermeidet zumindest die Falle, dass in $\operatorname{AT}_{\mathrm{E}} \mathrm{X}$ so ziemlich alles gut aussieht, und man sich auch selber täuschen kann.

Ja, wobei für mich der Grund ist, dass ich so langsam bin im Tippen und es so einfach effektiver ist.

Wobei man das ja lernen kann, aber ein Schreibmaschinenkurs war bisher nicht drin oder ist nicht geplant?

[lacht] Also der steht auf der langen Liste der Projekte, die ich immer mal machen will. Als ich Kind war oder Teenager, da schien mir das langweilig, da hab ich lieber Aufgaben der Mathe-Olympiade gemacht. Dass das theoretisch nützlich ist, das hat man mir schon erklärt, aber man glaubt ja seinen Eltern nicht. Und im Studium und in der Promotion war dafür keine Zeit ... Irgendwann hab ich mir mal so ein Programm runtergeladen und hab damit angefangen, bin aber nicht weit gekommen. Und seit ich ein Kind hab ist es einfach hoffnungslos ... [lacht]

Ja, wenn Du das als Defizit wahrnehmen würdest, dann könnte man das ja ändern...

Ja, aber die Menge an Zeit, die es braucht, über ein mathematisches Projekt nachzudenken und zu grübeln, ist einfach soviel größer, als die Zeit, die ich übers Tippen verliere, weil ich nur halb so schnell tippe wie andere Leute. Deshalb macht es für mich keinen so signifikanten Unterschied in der Gesamtzeit.

Wie schnell denkst du? Einstein soll ja langsam gewesen sein und war immer stolz darauf, dass er lange braucht. Wettbewerbsmathematik braucht ja eine gewisse Geschwindigkeit. Bist du besonders schnell im Problemlösen?

Ich denke, ich bin ungefähr mittel. Das Gute ist ja, dass man bei einer Mathematikolympiade-Klausur immerhin mehrere Stunden Zeit hat, man hat mehr als eine Stunde pro Aufgabe. Es ist nicht wie beim GRE-Test [Graduate Record Exam, Anm. d. Red.].

Wie war der? Es gibt da ja den Mathe- und den Sprachtest.

Ja. Der Mathe-Test wäre relativ einfach, wenn man je 5 Minuten pro Aufgabe hätte. Vom Schwierigkeitsgrad her so erstes oder zweites Studienjahr. Aber leider hat man nur zweieinhalb Minuten pro Aufgabe oder drei. Also ungefähr halb soviel Zeit wie man bräuchte. Und es geht dann darum, dass man schneller rechnen kann als die anderen.

Das heißt, du hattest Probleme mit dem GRE?

Nein. Ich habe sogar ganz gut abgeschnitten. Ich hab's schon vergessen, ich glaube über $97 \%$ der anderen Teilnehmer. Also schon ein ganz gutes Ergebnis, aber ich fand den Test einfach blöd.
Und die andere Hälfte ist dann ja ein Sprachtest, der aber für Muttersprachler gedacht ist, also der "Sprachliches Vermögen-Test" für alle, die in die graduate school wollen. Wenn man da besser ist als $80 \%$ der Amerikaner.

Also im Sprachteil war ich nicht bei $80 \%$, eher so bei $52 \%$.

Gut, aber das ist immer noch besser als die Hälfte der Amerikaner. Wie war denn dann sprachlich der Einstieg ins Studium in den USA?

Ach, das ging. In Bonn ist es so, dass die Masterkurse alle schon auf Englisch sind. Und ich hatte schon im BachelorStudium Masterkurse. Zum Teil sind auch im letzten Jahr die Bachelor-Vorlesungen auf Englisch. Insofern war das ein guter Übergang.

Wenn man sich jetzt mal so deine Karriere anguckt, also von den Schülerwettbewerben so ziemlich jeden gewonnen, dann Studium in Bonn und Promotion in Stanford und dann steht im Lebenslauf plötzlich ein Baby und ein Mann ... Ist das dann eine große Unterbrechung oder ist das einfach folgerichtig so?

Nach der Geburt unserer Tochter ging es mathematisch für mich natürlich schon etwas langsamer. Aber insgesamt ging es im Jahr nach der Geburt mathematisch sogar besser, als ich befürchtet hatte. Insofern war es eigentlich keine große Unterbrechung.

Wie viele Jahre waren das jetzt, für das Promotionsstudium in Standford?

Fünf Jahre. Das ist auch die normal übliche Dauer für das PhD-Programm in Stanford, weil mit dem PhDProgramm in gewissem Sinne Master und Promotion kombiniert werden. Meine Tochter wurde am Anfang des letzten Jahres geboren ...

Wie sieht es mit Vorbildern aus? Es sind ja in der Mathematik immer noch sehr viel weniger Frauen als Männer. Und es gibt diese leaky pipeline ... das heiß, der Frauenanteil geht mit jeder Karrierestufe runter. Und wenn man sich in Deutschland anschaut, welche Professorinnen Familie und Kinder haben, dann ist die Statistik noch viel schlechter. Hast du den Eindruck, du hast da genug Vorbilder? Oder ist das ein Kampf zu sagen, das ist mein Leben und das Kind gehört dazu?

Also die Leute in Stanford, also im Mathe Department in Stanford, die waren alle ganz toll, ganz super und sehr supportive, also ... unterstützend. Es hat dann auch ein Jahr gedauert, bis wir eine Kinderbetreuung für unsere Tochter hatten, das ist ja immer ein bisschen schwierig. Da haben wir unsere Tochter auch öfter mal mit ins Department gebracht zum Tee, nachmittags um halb vier, und die Leute haben sich alle immer gefreut unser Baby zu sehen und waren immer sehr unterstützend. Wir waren allerdings die einzigen Doktoranden mit Kind. Mein Mann ist gerade erst mit seiner Doktorarbeit fertig geworden, war also auch beim Promovieren, als unsere Tochter geboren wurde. 


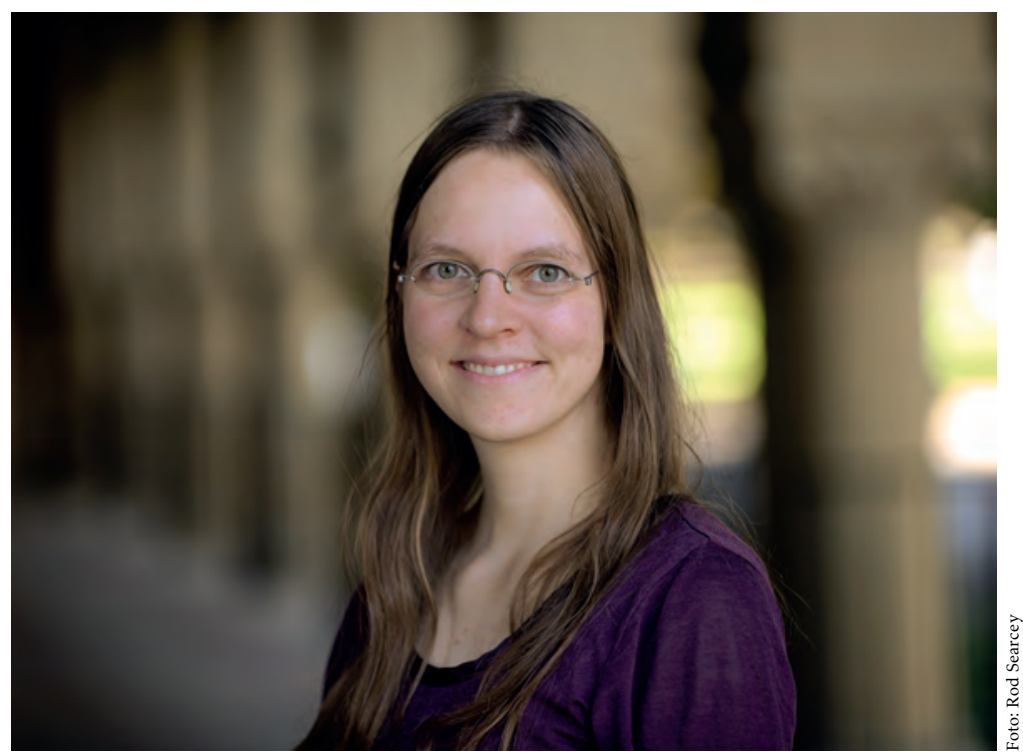

Lisa Sauermann in Stanford

Dein Mann hat in Differentialgeometrie promoviert, oder?

Ja, in symplektischer Geometrie. Weil wir die einzigen Doktoranden mit Kind waren, gab es jetzt keine besonderen Strukturen oder Pläne dazu, wie das MatheDepartment damit umgeht. Aber es waren alle sehr verständnisvoll und unsere Promotionsbetreuer waren auch beide super. Aber es stimmt, es gibt nicht so viele Vorbilder. Und die Entscheidung, während der Promotion ein Kind zu haben, ist vielleicht auch etwas ungewöhnlich und es gibt nicht so viele Leute, die das in den USA machen, in Mathe zumindest. Aber wir dachten uns, es ist ein guter Zeitpunkt, weil ich ein Stipendium hatte und nicht unterrichten musste und einige Projekte für meine Dissertation schon fertig hatte. Das passte gut und ich denke das war wirklich der richtige Zeitpunkt.

Es gibt immer noch wenige Frauen in der Mathematik und zurück in die Geschichte geschaut noch weniger. Gibt es Leute, von denen du dir lange gesagt hast, also eigentlich will ich in die und die Richtung?

Es gibt jetzt keine konkrete Person. Aber verschiedene weibliche Forscherinnen auf Konferenzen zu sehen, und zu sehen, dass es die auch gibt, das hat schon geholfen. Auch wenn ich da jetzt keine einzelne Person herausheben kann, denke ich, dass das schon Eindruck hinterlassen hat.

Noch zum Thema Karriere: Die Leute werden in Deutschland fragen, ob du vielleicht wieder zurückkommst...

Also ab nächstem Jahr sind wir erst mal am IAS [Institute for Advanced Study, Princeton, Anm. D. Red.].

Beide gemeinsam? Wunderbar!
Ja, wir haben dort Postdoc-Stellen für ein Jahr. Und dann geht's für mich weiter ans M.I.T. auf eine tenure trackPosition.

Großartig, Glückwunsch! Und dein Mann ...?

Der hat eine Postdoc-Stelle in Harvard, während ich die tenure track-Stelle am M.I.T. habe.

Das ist ja perfekt! Und die Karriere ist zumindest für die nächsten Jahre auch erst mal gesichert ...

Ja, da haben wir echt Glück gehabt. Aber das ist ja erst mal nur tenure track und vom M.I.T. ist bekannt, dass es tenure-Raten hat, die nicht an $100 \%$ grenzen, sondern weit darunter liegen, so bei $30 \%$.

In der Mathematik in Princeton waren sie mal nah bei o \%.

Insofern glaube ich nicht, dass ich da auf jeden Fall tenure [eine Festanstellung, Anm. d. Red.] bekomme. Auch mein Mann hat erst einmal nur eine Postdoc-Stelle für vier Jahre. Eventuell müssen wir uns also danach noch einmal umsehen, und hoffentlich ergibt sich dann was.

Ist die momentane politische Situation eigentlich ein Thema bei euch?

Auf jeden Fall. Die Visa-Situation ändert sich ja auch ständig. Im Moment werden die Regeln durch die Pandemie begründet. Aber falls Trump wiedergewählt wird, ist es nicht unmöglich, dass wir aus Visa-Gründen hier in den USA Probleme kriegen. Aber wir lassen das alles in Ruhe auf uns zukommen.

\section{Welcher Nationalität ist dein Mann?}

Der ist Kanadier. Der hat im Wesentlichen dieselben Probleme wie ich. Manche bürokratische Prozesse sind für Kanadier etwas einfacher. Aber im Wesentlichen ist das alles das gleiche. 
Ja, das ist eine verrückte Welt ... Das waren jetzt die Karriere-Herausforderungen. Was ist mit den mathematischen? Nimmt man da vielleicht noch mal Anlauf auf eines der ganz großen Probleme? $P=N P$ oder was Vergleichbares?

Nee, also $P=N P$ liegt nicht wirklich in meinem Gebiet. Millenniumsprobleme auf keinen Fall, große Probleme in der Kombinatorik schon eher. Also den Rat, den ich von meinem Betreuer und auch anderen gehört habe, ist der: Es ist gut, die großen Probleme zu kennen, falls man doch mal irgendwie ne Idee hat, also, dass man Bescheid weiß. Aber es ist für mich nicht so, dass ich größere Probleme in extremaler Kombinatorik lösen muss. Wichtiger ist, dass es mir Spaß macht und dass ich interessant finde, was ich mache...

Was wäre so das größte Problem in der Kombinatorik? Asymptotik für Ramsey-Zahlen?

Ja, das ist auf alle Fälle eines der großen! Ich weiß nicht wirklich ob man sagen kann, das und das ist das größte. Aber wenn man eine Shortlist von fünf macht, dann wäre das auf jeden Fall dabei.

Also für den Helden in „Nagel im Himmel“ geht es um die Riemann-Hypothese...

Nein, das ist eher nichts für mich! [lacht]

Also, we ,covered quite some ground', wie auch immer das auf Deutsch heißt. Was macht dein Deutsch, rostet es langsam ein?

Also ich glaube, es geht noch, jedenfalls wenn man von „supportive“ vorhin absieht. Ich lese die Nachrichten immer auf Deutsch, damit mein Deutsch nicht einrostet, und ich unterhalte mich mit meinen Eltern regelmäßig über Skype ... und dann rede ich mit meiner Tochter Deutsch. Die redet zwar noch nicht so viel zurück, aber ...

Wie alt ist die jetzt?

Knapp zwei.

Mit dem Papa redet sie dann Französisch?

Genau. Das ist übrigens auch auf meiner Liste der zu lernenden Dinge, wie mit zehn Fingern tippen zu lernen, also besser Französisch zu lernen. Aber da müsste ich auch einfach viel mehr Zeit investieren, sonst wird das nichts. [lacht]
Da hilft natürlich, wenn man einen netten Privatlehrer hat...

Ja, aber man muss trotzdem Zeit investieren ...

Ja genau, Zeit investieren wird dann eher das Problem sein, da hat man keine Zeit für weitere Hobbys ...

Nein, neben dem Spielen mit meiner Tochter Clara ist dann leider keine Zeit mehr für andere Freizeitaktivitäten. Vor allem jetzt in der Pandemie, die Kinderbetreuung ist ja immer noch zu. - Aber wir haben vor zwei Tagen die große Neuigkeit bekommen, dass es in eineinhalb Wochen mit der Kinderbetreuung wieder losgehen kann. Die Kinderbetreuung ist vier Monate zu gewesen.

Und das hat dann auch das Mathematikmachen ein bisschen entschleunigt?

Ja, auf jeden Fall. Mein Mann und ich haben uns abgewechselt, auf Clara aufzupassen, aber dann hat man natürlich nur noch halb so viel Zeit für Mathe.

Wenn du so ein mathematisches Problem mit dir rumträgst, in welchen Situationen hast du dann am ehesten eine gute Idee?

In allen möglichen Situationen: manchmal beim Einschlafen, manchmal beim Duschen, letztens sogar mal, als ich mit meiner Tochter gespielt habe, aber das ist eher selten, denn da kann man sich nicht so richtig konzentrieren. Manchmal natürlich auch ganz klassisch am Schreibtisch sitzend beim Nachdenken. In allen möglichen Situationen.

Aber kommen die Ideen vom Konzentrieren? Oder kommen die Ideen, weil die Sachen einem im Kopf herumschwirren? Und dann kommen die Sachen zusammen, wenn man mit der Tochter spielt?

Ja, das kann natürlich gut sein. Es kann sein, dass man zwei Stunden am Schreibtisch sitzt und nachdenkt an einem Tag und dann am nächsten Morgen, wenn man etwas anderes macht, sich die Idee zusammenfügt. Ja, ich denke schon, dass es so einen Effekt gibt.

Das Online-Interview führte Günter M. Ziegler, Jahrgang 1963, IMO-Goldmedaille 1981, später Herausgeber der Mitteilungen und Präsident der DMV, jetzt Präsident der Freien Universität Berlin, im Juli 2020. 